\title{
Simple $\beta$-lactones are potent irreversible antagonists for strigolactone receptors
}

Cell Research (2017) 27:1525-1528. doi:10.1038/cr.2017.105; published online 18 August 2017

\section{Dear Editor,}

Strigolactones (SLs) are a class of plant hormones that play vital roles not only in the control of plant branching and early seedling growth, but also in mediating rhizospheric communication with root parasitic plants and symbiotic fungi [1]. SL receptors in host and parasitic plants have recently been identified to be a group of $\alpha / \beta$ hydrolases [2-7], but not in arbuscular mycorrhizal fungi, which form symbiosis with more than $80 \%$ of land plants [8].

Small-molecule inhibitors of SL signaling could be valuable research tools as well as lead compounds for agrochemical development to eradicate root parasitic plants. In this regard, a triazole-containing small molecule has been reported to inhibit the biosynthesis of SLs [9]. Recently, McCourt and co-workers [10] have reported non-covalent antagonists of SL receptors with micromolar $\mathrm{IC}_{50} \mathrm{~s}$ by using small-molecule high-throughput screening. Based on the mechanistic understanding of SL perception $[2,3]$, here we developed a group of $\beta$-lactones as potent covalent antagonists for strigolactone receptors with nanomolar $\mathrm{IC}_{50} \mathrm{~s}$.

The serine residue of the catalytic triad Ser-His-Asp in SL receptors attacks the carbonyl group of the SL D-ring to initiate the hydrolysis, resulting in a His covalently linked intermediate molecule that activates downstream signaling. We hypothesized that $\beta$-lactones, with the ring strain energy around $20 \mathrm{kcal} / \mathrm{mol}$, could readily react with the conserved serine residue in the active site and thus block the perception of SL (Figure 1H). Given the variation in the active sites of SL receptors in host and parasitic plants $[4,5]$, which is also reflected by the different bioactivities of SLs $[5,6]$, we envisioned that the potency and selectivity of these perspective inhibitors would be fine-tuned by substituents and their stereochemistry.

To probe this hypothesis, we synthesized six $c i s-\beta$-lactones (Figure 1A) using the enantioselective ketene-aldehyde cyclocondensation reactions catalyzed by asymmetric cinchona alkaloids [11]. This method developed by the Nelson group enabled the efficient preparation of both enantiomers of $c i s-\beta$-lactones, the enantiopurity of which was determined by chiral-HPLC (Supplementary information, Data S1). The biological function of SL receptors is dependent on the hydrolase activity, which could be readily measured using a profluorescent probe, Yoshimulactone green (YLG) [6]. We therefore established this biochemical assay using AtD14 as the representative receptor that perceives SLs as a plant hormone, and ShHTL7 that shows the highest activity among Striga SL receptors [5]. The apparent $K_{\mathrm{m}}$ (YLG) values of AtD14 and ShHTL7 were determined to be 0.47 and 2.4 $\mu \mathrm{M}$ respectively, which were consistent with those in the literature [6] (Supplementary information, Figure S1). The $\mathrm{IC}_{50}$ values of synthesized cis- $\beta$-lactones and a trans$\beta$-lactone, Palmostatin B (a known acyl protein thioesterase inhibitor) [12] were determined under either 1 or 5 $\mu \mathrm{M}$ YLG concentrations (Figure 1B and Supplementary information, Figure S2).

To our delight, TFQ0010 turned out to be a very potent inhibitor of AtD14, while the potency of its enantiomer, TFQ0011, was a magnitude lower, suggesting a specific chiral environment of the binding pocket is involved. Similar stereoselectivity of AtD14 towards its agonists has been reported in previous studies [13, 14]. Replacing the phenylethyl side chain on $\mathrm{C} 4$ with a phenyl group (TFQ0020) significantly reduced the potency, but replacing the methyl group on $\mathrm{C} 3$ with ethyl (TFQ0021) and propyl group (TFQ0022) conferred three- and four-fold improvement in potency, respectively. Notably, similar potency was obtained in TFQ0023 with a propargyl group on $\mathrm{C} 3$, which provides a handle for bioorthogonal conjugation. Interestingly, Palmostatin $\mathrm{B}$, as a trans- $\beta$-lactone with the $3 S, 4 S$ stereochemistry and a much longer substituent on $\mathrm{C} 3$, could also potently inhibit the hydrolase activity of AtD14. In comparison, the structure-activity relationship of $\beta$-lactones in inhibiting ShHTL7 revealed a totally different trend. Only TFQ0011 with the $3 S, 4 R$ stereochemistry inhibited the hydrolase activity of ShHTL7 with submicromolar $\mathrm{IC}_{50}$. This observation, together with the $\mathrm{IC}_{50}$ of Palmostatin 
A

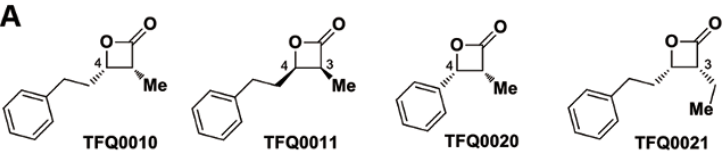<smiles>CCCC(=O)OCc1ccccc1</smiles>

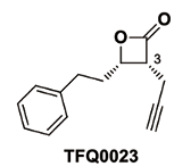

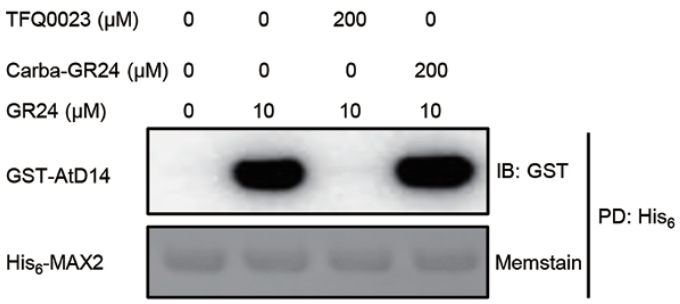

$\mathbf{F}$

\begin{tabular}{|c|c|c|c|c|}
\hline \multirow{2}{*}{$\frac{\beta \text {-lactones }}{\text { TFQ0010 }}$} & \multicolumn{2}{|c|}{$\begin{array}{l}I C_{50} \text { for AtD14 }(\mu \mathrm{M}) \\
1 \mu \mathrm{M} \text { YLG } 5 \mu \mathrm{M} \text { YLG }\end{array}$} & \multicolumn{2}{|c|}{$\begin{array}{c}\left.\mathrm{IC}_{50} \text { for ShHTL7 ( } \mu \mathrm{M}\right) \\
1 \mu \mathrm{M} \text { YLG }\end{array}$} \\
\hline & $0.678 \pm 0.037$ & $0.573 \pm 0.043$ & $55.8 \pm 3.6$ & $77.2 \pm 20.9$ \\
\hline TFQ0011 & $7.59 \pm 0.42$ & $7.89 \pm 0.87$ & $0.472 \pm 0.087$ & $0.923 \pm 0.043$ \\
\hline TFQ0020 & $>100$ & N.D. & $27.4 \pm 3.7$ & $37.8 \pm 14.1$ \\
\hline TFQ0021 & $0.209 \pm 0.009$ & $0.163 \pm 0.010$ & $7.05 \pm 0.54$ & $9.31 \pm 4.20$ \\
\hline TFQ0022 & $0.174 \pm 0.010$ & $0.157 \pm 0.008$ & $19.2 \pm 1.1$ & $27.3 \pm 6.4$ \\
\hline TFQ0023 & $0.411 \pm 0.020$ & $0.446 \pm 0.042$ & $23.2 \pm 1.2$ & $14.2 \pm 1.1$ \\
\hline Palmostatin B & $0.245 \pm 0.010$ & $0.286 \pm 0.025$ & $1.01 \pm 0.07$ & $1.39 \pm 0.42$ \\
\hline
\end{tabular}

C
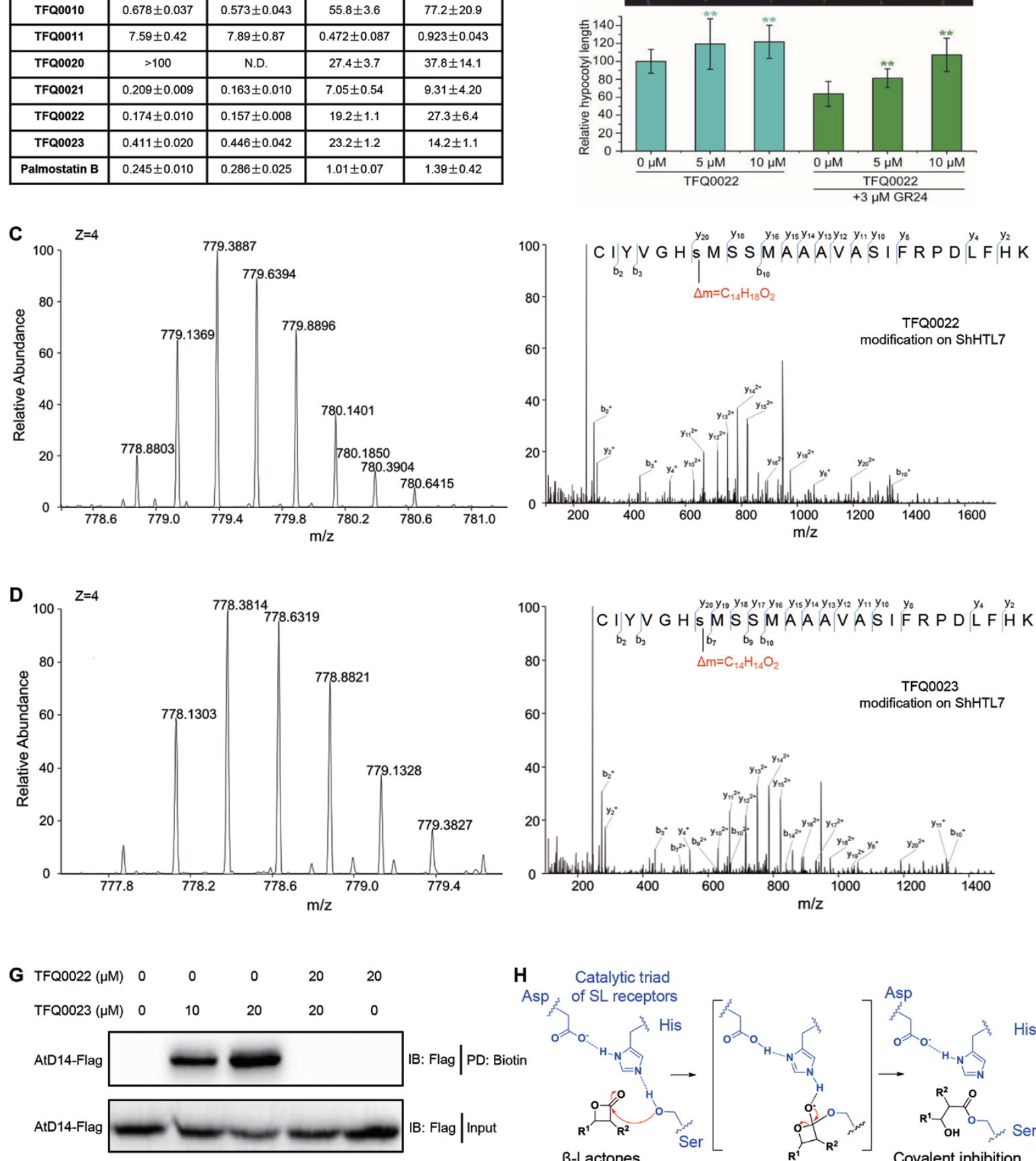

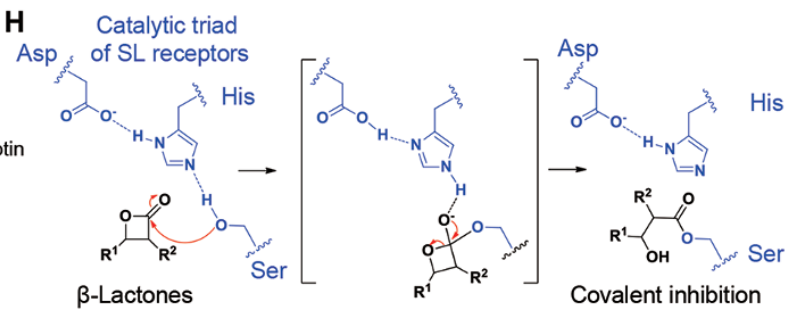


B around $1 \mu \mathrm{M}$, argued for the preference of ShHTL7 toward $\beta$-lactones with the $3 S$ stereochemistry, which could be exploited to develop more selective inhibitors for Striga SL receptors. For all inhibitors, similar $\mathrm{IC}_{50}$ values were obtained using 1 and $5 \mu \mathrm{M}$ YLG for both AtD14 and ShHTL7, which was consistent with the irreversible inhibitor hypothesis. As a preliminary counter screening to evaluate the selectivity of these $\beta$-lactones, we measured their inhibitory activities against another $\alpha /$ $\beta$ hydrolase, salicylic acid binding protein 2 (SABP2), which was used as a negative control in the SL hydrolysis assay [15]. The $\mathrm{IC}_{50}$ of TFQ0010 turned out to be 25.3 $\mu \mathrm{M}$, demonstrating a $\sim 40$-fold selectivity for AtD14 over $\mathrm{SABP} 2$, whereas the $\mathrm{IC}_{50}$ values of all the other tested $\beta$-lactones were over $300 \mu \mathrm{M}$ (Supplementary information, Table S1).

To further elucidate the inhibitory mechanism of $\beta$-lactones, we employed liquid chromatography-tandem mass spectrometry (LC-MS/MS) to investigate the covalent modification of SL receptors by these putative irreversible inhibitors. As expected, the covalent modifications on Ser95 of ShHTL7 were readily detected after incubating ShHTL7 with the inhibitors TFQ0022 (Figure 1C) and TFQ0023 (Figure 1D), unequivocally confirming the stable acylation of the conserved serine in the catalytic triad by $\beta$-lactones (Figure $1 \mathrm{H}$ ). Similarly, the modifications were also successfully detected on Ser97 of AtD14 (Supplementary information, Figure S3).

To investigate whether the blockage of Ser95 by $\beta$-lactones is sufficient to prevent the interaction of the SL receptor with interacting partners, we set up a competitive pull-down assay to examine the influence of TFQ0023 on SL-induced AtD14-MAX2 interaction. As shown in
Figure 1E, TFQ0023 effectively inhibited the SL analog GR24-induced AtD14 interaction with MAX2, while an inactive SL mimic Carba-GR24 did not. These results suggest that these $\beta$-lactones may have the capacity to impede the SL signaling pathway in planta. Indeed, the application of TFQ0022 on wild-type Arabidopsis seedlings (Col-0) relieved the SL repression on hypocotyl elongation not only in the absence of GR24 but also in the presence of GR24 (Figure 1F). These results also indicate that TFQ0022 may have the capacity to outcompete the endogenous SLs.

Moreover, the irreversible inhibitors developed by us could be used to isolate SL receptors. We validated this approach by using TFQ0023 to label and pull down the Arabidopsis SL receptor AtD14 from the total plant extracts (Figure $1 \mathrm{G}$ ). Total extracts of $35 \mathrm{~S}$ promoter-driven AtD14-Flag transgenic plants were incubated with TFQ0023 at room temperature, after which the TFQ0023-labeled proteins were denatured and subjected to subsequent click reaction for tagging the terminal alkyne in TFQ0023 with an azide-biotin. Magnetic streptavidin beads were then used to enrich the biotin-tagged TFQ0023 and its associated proteins for further western blot analysis. The results shown in Figure $1 \mathrm{G}$ indicate that the SL receptor AtD14 in crude plant extracts could be successfully labeled and pulled down by TFQ0023, and the reaction could be inhibited by the addition of a more potent $\beta$-lactone antagonist, TFQ0022.

In conclusion, we have developed potent irreversible inhibitors of SL receptors on the basis of SL perception mechanism $[2,3]$ and the structural similarity of $\beta$-lactones and the D-ring of SLs. In comparison to the prior

Figure 1 (A) Chemical structures of $\beta$-lactones evaluated in this report. (B) Biochemical activity of $\beta$-lactones in inhibiting YLG hydrolysis by the SL receptors AtD14 and ShHTL7. Corresponding curves were shown in Supplementary information, Figure S2. (C, D) $\beta$-Lactones covalently modify Ser95 of the catalytic triad in the SL receptor ShHTL7. (C) The MS spectrum of a quadruply charged ShHTL7 peptide at mass-to-charge ratio $(\mathrm{m} / \mathrm{z}) 779.3887$ (left panel) corresponding to the TFQ0022 modification on Ser95 (right panel) of the peptide. (D) The MS spectrum of a quadruply charged ShHTL7 peptide at $\mathrm{m} / \mathrm{z} 778.3814$ (left panel) corresponding to the TFQ0023 modification on Ser95 (right panel) of the peptide. Carbamidomethylation on Cys was carried out. The ShHTL7 peptides were produced by trypsin digestion. (E) $\beta$-Lactone inhibited the SL-induced AtD14MAX2 interaction. Pull-down assays were carried out using recombinant His ${ }_{6}-\mathrm{MAX} 2$ and GST-AtD14 in the absence or presence of GR24, Carba-GR24 and TFQ0023. The pulled down GST-AtD14 was detected by anti-GST antibody and the PVDF

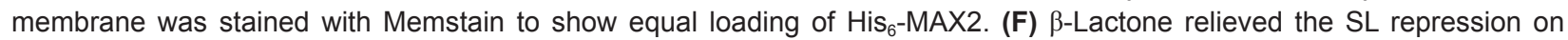
hypocotyl elongation. Representative hypocotyl phenotype (upper panel) and relative hypocotyl lengths (lower panel) of wildtype Arabidopsis Col-0 under the treatment with indicated chemicals. Scale bar, $5 \mathrm{~mm}$. Data are means $\pm \mathrm{sd}(n=40$ plants per treatment); ${ }^{* *} P<0.01$ (t-test). (G) TFQ0023 efficiently targeted and modified AtD14 in plant extracts. AtD14-Flag transgenic plant extracts were treated with TFQ0023 in the absence or presence of competitor TFQ0022, and subsequent click reaction was carried out to attach the biotin tag on TFQ0023. After purification through the biotin tag (PD: Biotin), the enriched AtD14-Flag proteins were detected by anti-Flag antibody (upper panel, western blotting against Flag tag, i.e., IB: Flag). Input represents one percent of the plant extracts used for each set of trial (detected by anti-Flag antibody as shown in the lower panel). (H) A proposed mechanism of action of $\beta$-lactones. Acylation of the serine residue within the catalytic triad (Ser-His-Asp) in the active site leads to the inhibition of SL receptors. 
art (soporidine) that targets AtHTL and ShHTL7 [10], this group of rationally designed $\beta$-lactones can act as potent antagonists for AtD14 and ShHTL7 and their mode of inhibition involves irreversible attachment to the conserved serine of the catalytic triad. Unlike the butenolides in SLs and SL mimetics, the reaction of strained $\beta$-lactones with AtD14 does not lead to receptor activation. The divergent structure-activity relationship of these compounds toward AtD14 and ShHTL7 implies that selective inhibition of Striga germination while sparing the SL receptors in host plants is possible. This possibility could be further strengthened by the variation in the active sites of SL receptors in host and parasitic plants: the ligand binding pockets of weed SL receptors are much larger than that of the rice or Arabidopsis receptor, and the key amino acids in the pockets differ from each other to some extent $[4,5]$. Given the advantage of prolonged duration of inhibition provided by this kind of covalent inhibitors and the ease of synthesis, the preparation of a focus library of $\beta$-lactones is warranted to further optimize this series of inhibitors for field trials. Furthermore, a proof-of-concept study labeling the SL receptor AtD14 in Arabidopsis suggests a new approach to identify SL receptors by means of the development of small-molecule probes in combination with activity-based proteome profiling. We hope that these readily prepared compounds will help researchers in the strigolactone community to further elucidate the biology of this fascinating family of plant hormones and develop agricultural applications in the near future.

\section{Acknowledgments}

We apologize that we are unable to cite many excellent original works due to space limit. This work was supported by generous start-up funds from the National "Young 1000 Talent Plan" Program, College of Chemistry and Molecular Engineering, Peking University, Peking-Tsinghua Center for Life Sciences, the National Natural Science Foundation of China (Grant Nos. 31630085, 21472003 and 21672011), the Ministry of Science and Technology of China (Grant No. 2016YFA0500501), Tsinghua-Peking Joint Center for Life Sciences (a postdoctoral grant for RY) and China Association for Science and Technology (a grant for RY). We thank Professor Jian Wang (Tsinghua University) for his help in chiral HPLC analysis.
Haibo Xiang ${ }^{1,}$,, Ruifeng Yao ${ }^{2, *}$, Tianfei Quan ${ }^{1}$, Fei Wang', Li Chen ${ }^{2}$, Xiaoxi Du², Wenhao Zhang ${ }^{2}$, Haiteng Deng ${ }^{2}$, Daoxin $\mathrm{Xie}^{2}$, Tuoping Luo ${ }^{1}$

${ }^{I}$ Peking-Tsinghua Center for Life Sciences, Academy for Advanced Interdisciplinary Studies, Key Laboratory of Bioorganic Chemistry and Molecular Engineering, Ministry of Education, Beijing National Laboratory for Molecular Science, College of Chemistry and Molecular Engineering, Peking University, Beijing 100871, China; ${ }^{2}$ Tsinghua-Peking Joint Center for Life Sciences, and MOE Key Laboratory of Bioinformatics, School of Life Sciences, Tsinghua University, Beijing 100084, China

*These two authors contributed equally to this work.

Correspondence: Tuoping Luo ${ }^{\mathrm{a}}$, Daoxin $\mathrm{Xie}^{\mathrm{b}}$

aE-mail: tuopingluo@pku.edu.cn

bE-mail: daoxinlab@mail.tsinghua.edu.cn

\section{References}

1 Waters MT, Gutjahr C, Bennett T, et al. Annu Rev Plant Biol 2017; 68:291-322.

2 Yao R, Ming Z, Yan L, et al. Nature 2016; 536:469-473.

3 Yao R, Wang F, Ming Z, et al. Cell Res 2017; 27:838-841.

4 Conn CE, Bythell-Douglas R, Neumann D, et al. Science 2015; 349:540-543.

5 Toh S, Holbrook-Smith D, Stogios PJ, et al. Science 2015; 350:203-207.

6 Tsuchiya Y, Yoshimura M, Sato Y, et al. Science 2015; 349:864-868.

7 Hamiaux C, Drummond RS, Janssen BJ, et al. Curr Biol 2012; 22:2032-2036.

8 Gutjahr C, Gobbato E, Choi J, et al. Science 2015; 350:15211524.

9 Ito S, Kitahata N, Umehara M, et al. Plant Cell Physiol 2010; 51:1143-1150.

10 Holbrook-Smith D, Toh S, Tsuchiya Y, et al. Nat Chem Biol 2016; 12:724-729.

11 Zhu C, Shen X, Nelson SG. J Am Chem Soc 2004; 126:53525353.

12 Rusch M, Zimmermann TJ, Burger M, et al. Angew Chem Int Ed Engl 2011; 50:9838-9842.

13 Scaffidi A, Waters MT, Sun YK et al. Plant Physiol 2014; 165:1221-1232.

14 Umehara M, Cao M, Akiyama K, et al. Plant Cell Physiol 2015; 56:1059-1072.

15 Zhao LH, Zhou XE, Wu ZS, et al. Cell Res 2013; 23:436-439.

(Supplementary information is linked to the online version of the paper on the Cell Research website.) 